\title{
Causes of Psychological Reactance in Human-Computer Interaction
}

\author{
A Literature Review and Survey
}

\author{
Patrick Ehrenbrink \\ Institute of Software Engineering and Theoretical \\ Computer Science \\ Quality and Usability Lab \\ Technische Universitt Berlin \\ Ernst-Reuter-Platz 7 \\ Berlin, Germany 10587 \\ patrick.ehrenbrink@tu-berlin.de
}

\author{
Sabine Prezenski \\ Mechanical Engineering and Transport Systems \\ Cognitive Modeling in dynamic Human-Machine \\ Systems \\ Technische Universitt Berlin \\ Marchstr. 23 \\ Berlin, Germany 10587 \\ sabine.prezenski@tu-berlin.de
}

\begin{abstract}
Psychological reactance is a psychological effect that can reduce the acceptance of devices and services. So far, there has not been a collection of potential causes for reactance in the context of human-computer interaction in literature. A literature review and a qualitative study to compile a list of possible causes of reactance in human-computer interaction were conducted. The final list includes twelve causes of reactance that have been shown to appear in the field of human-computer interaction. The individual causes are discussed and examples provided. Finally, strategies for avoiding reactance in human-computer interaction are proposed and possible future directions for reactance research in this field are recommended.
\end{abstract}

\section{CCS CONCEPTS}

- Human-centered computing $\rightarrow$ Empirical studies in interaction design; HCI theory, concepts and models; Interaction design theory, concepts and paradigms;

\section{KEYWORDS}

Psychological Reactance, Human Computer Interaction, Acceptance

ACM Reference format:

Patrick Ehrenbrink and Sabine Prezenski. 2017. Causes of Psychological Reactance in Human-Computer Interaction. In Proceedings of ECCE 2017, Umeå, Sweden, September 19-22, 2017, 8 pages. https://doi.org/10.1145/3121283.3121304

\section{INTRODUCTION}

Psychological Reactance is a construct that has its origins in social psychology. In 1966, Brehm published his book "A

Permission to make digital or hard copies of part or all of this work for personal or classroom use is granted without fee provided that copies are not made or distributed for profit or commercial advantage and that copies bear this notice and the full citation on the first page Copyrights for third-party components of this work must be honored. For all other uses, contact the owner/author(s).

ECCE 2017, September 19-22, 2017, Umeå, Sweden

(C) 2017 Copyright held by the owner/author(s).

ACM ISBN 978-1-4503-5256-7/17/09.

https://doi.org/10.1145/3121283.3121304
Theory of Psychological Reactance" which introduced and discussed the concept in detail [1]. There is a famous internet meme from 2006, called "Angry German Kid". It originates from a video that shows a young boy sitting at his desk and wanting to play a computer game. However, first the game is loading quite slowly, then the boy is actually losing the game. A screenshot from the video can be seen in Figure 1. During the whole video, the boy gets increasingly angry and starts shouting and abusing his keyboard until he destroys it in the end and runs away. While the video itself is most likely staged, it provides a good impression of exaggerated psychological reactance. According to Brehm, reactance is a motivational state that a person enters when she or he perceives a threat to freedom or control. That person will then engage in countermeasures to reestablish the supposedly lost freedom or control $[1,2]$. In the video, the boy wants to play a computer game but is hindered from doing so by the fact that the computer is loading the game too slowly. He then enters a reactant state and starts shouting at the computer and even abuses the keyboard. The boy's violence against the computer can be interpreted as reactant behavior. The computer poses a threat to the boy's freedom and control over the situation. Therefore, the boy attacks the computer in an attempt to eliminate the freedom threat. The boy's extreme expression of anger and shouting is also consistent with more recent findings of reactance research. In 2005, Dillard and Shen introduced the intertwined model of psychological reactance [3]. According to that model, reactance is a construct that consists of anger and negative cognitions, both entangled in a way that the individual contribution on the level of reactance cannot be explained [3]. In that video, the boy obviously gets angry towards his computer. Also, negative cognitions are apparent, considering the amount of swear words and insults that he shouts at his monitor. A similar, but probably not that extreme reaction can also occur in other parts of humancomputer interaction. The phenomenon of reactance has been described in human-computer interaction literature several times [4-6, 11-17], but so far there is no general overview on how and when reactance usually takes place in HCI. This is even though reactance can have severe consequences on user acceptance, up to the complete inversion of the desired effect, 


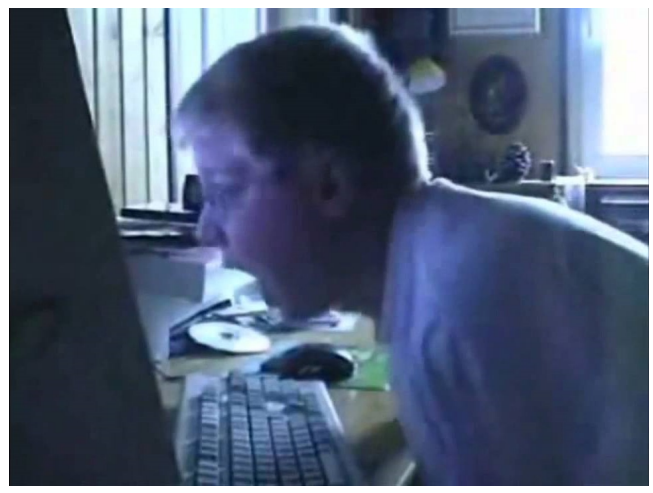

Figure 1: Screenshot of a video that went viral in 2006 [9]

which has already been demonstrated for persuasive health campaigns [3]. For HCI, at least a diminished acceptability has been shown to correlate with reactance [5]. In this work, a summary of the appearances of reactance in human-computer interaction research and an overview of the identified causes of reactance is provided. Furthermore, this information is enhanced with a qualitative survey conducted among humancomputer interaction experts.

\section{REACTANCE IN LITERATURE}

Search on Google Scholar [7] and references from literature to identify relevant literature from the field of human-computer interaction. As the aim of this paper is to provide an overview of the sources of reactance in human-computer interaction and not a complete literature review, completeness for either the reactance sources or the relevant literature is not guaranteed. In total, ten papers were found that empirically investigated reactance or employed reactance as an explanation for empirical findings in the field of human-computer interaction. Papers not including empirical results were not considered. Also, there are several papers from marketing research that discuss reactance, mostly as an intervening variable. Some of such papers were considered because they discussed reactance in the context of e-mails or websites. In the following, we will list the causes of reactance that we identified. A list of those causes is also provided in Table 1.

Table 1: Clusteres and frequency of different causes for reactance that were identified in literature.

\begin{tabular}{lc}
\hline Cause & Frequency \\
\hline Persuasive Attepts & 3 \\
Privacy Violations & 2 \\
Reduced Freedom of Choice & 1 \\
System Errors & 1 \\
Self Adaptation & 1 \\
Forced Response & 1 \\
Obstructions & 1 \\
\hline
\end{tabular}

\subsection{Persuasive Attempts}

We found three papers that discussed reactance as a result of persuasive attempts, triggered either by a smart home device [11] or by artificial agents $[13,15]$. Laschke et al. introduced a key holder that functions as a persuasive device. It is meant to persuade people to use the bicycle, instead of the car. If a person takes the car key, the key holder will drop the bicycle key to the floor, thereby trying to persuade the user to use the bicycle instead of the car [11]. The authors mention that interacting with their key holder could create friction, which could then result in reactance. They also propose to implement understanding, naive or ironic features to decrease or prevent reactance [11]. Liu et al. investigated the usefulness of artificial social agents in persuasive systems [13]. They used a two by two conditional design. One factor was task- or agent-oriented decision making. Where agent-oriented decision making emphasized agreement with the agent and task-oriented decision making did not. The other condition was a persuasive vs. non-persuasive condition. They found reactance effects when participants decide to agree or disagree with an agent. Even when participants could have significantly reduced their workload and receive a 100 percent correct result simply by always agreeing with the artificial agent, some choose the disagree option [13]. Liu et al. found that the agent-oriented group performed significantly lower compared to the task-oriented group and that the difference between the groups was stronger in the persuasive condition than in the non-persuasive condition [13]. Roubroeks et al. investigated how social agency and controlling language of a persuasive system influences reactance of the users. They used a persuasive system in a three by three conditional design, where they varied the controlling language (non-controlling language, low controlling language, high controlling language) and the social agency of an accompanying agent (none, still image of a cat robot, video of a cat robot) [15]. What they found was that the level of reactance rises with higher controlling language throughout all agency conditions. Social agency did not produce such a clear effect, however reactance scores for the high social agency condition were significantly higher than the reactance scores of the low social agency condition [15].

\subsection{Privacy Violations}

Two of the papers that investigated reactance effects found that personalization [17] or collecting personal information [12] can be a reason why users might get reactant. White et al. investigated the influence of the personalized messages on reactance. Therefore, they conducted two studies with a two by two conditional design. One factor was highly distinctive personalization vs. less distinctive personalization in messages. The other factor was justification, where the personalization was either justified or not [17]. They found that highly distinctive personalization can cause more reactance than less distinctive personalization if personalization is not justified. In the condition with justified personalization, they did not find an effect of personalization on reactance. White 
et al. claim that reactance to personalization occurs when users get the impression of being identifiable or observable by the sender (a company), but an adequate justification for the personalization, e.g. where the information comes from, did not produce significantly higher reactance [17]. Lee and Lee investigated whether online recommendation services can cause psychological reactance. They argued, that recommendation by such services could be regarded restrictive or that users could feel pressured into accepting the recommendations, what would result in reactance [12]. They used a two conditional design for their experiment. In a high threat group, the users were told that the recommendation service would use a variety of personal information of the users for the recommendations. In the low threat group, the recommendation services used considerable less personal information. They found that the users of the high threat group expressed less intention to use the service again, compared to the users of the low threat group and interpreted those findings as evidence of reactance caused by the collection of personal data by the recommendation service [12].

\subsection{Self-Adaptation}

Self-adaptation of devices and services is often regarded as a positive functionality that is intended to increase the ease of use. However, users are used to be in control over their devices and handing over parts of that control to the device itself could be perceived as a loss of control which then could trigger psychological reactance. Lee and Lee postulated a similar hypothesis in their study that was discussed earlier [12]. Another study that investigated reactance effects in the context of adaptive system behavior was published by Ehrenbrink et al. [6]. They conducted and experiment where participants had to interact with a smart home system via a spoken dialogue system. In one condition, that system adapted automatically to the user, whereas in the other condition, the system had to be adapted manually by the user. Ehrenbrink et al. found that one component of reactance did not decrease (pre-measure minus post-measure) during interaction with the self-adaptive system but decreased during interaction with the user-adaptable system [6]. The results can be interpreted as evidence that adaptive system behavior could potentially result in reactance under some circumstances.

\subsection{Forced Response}

We found one paper that investigated reactance because of forced response in online surveys. Stieger et al. conducted an online study in which they implemented a forced response function in an online questionnaire. If participants did not answer one or more items, they could not proceed with the study. If participants were trying to skip the questionnaire, they were directed to a webpage that requested to complete the questionnaire [16]. Stieger et al. then investigated the dropout rates of those participants who had not tried to skip items or whole questionnaires, hence did not know that there was a forced response function and those participants who had tried to skip items or whole questionnaires, hence knew that there was a forced response function. What they found was that the dropout rates of those participants who knew that a forced response function was active was significantly higher compared to those participants who did not know about the forced response function [16]. This led Stieger et al. to the conclusion that forced response can cause reactance effects, such as dropping out of an online survey [16].

\subsection{System Errors}

System errors can be a source of reactance, especially if they result in undesired system behavior. When a system error occurs, providing feedback is a vital function of user interfaces because it adds to the situation awareness of the user and can provide hints toward the source of the error. If there is no or inadequate feedback provided by a user interface, then users could become reactant because not knowing about the source of an error can render users quite helpless. Ehrenbrink et al. conducted an experiment where participants interacted with a smart TV in three different conditions to investigate the implications of different types of feedback for user reactance. The smart TV could be controlled via voice commands and provided feedback for various levels of the processing chain of the voice commands. Not surprisingly, Ehrenbrink et al. found that reactance was higher if the system made errors, compared to when the system did not make errors [5]. What they also found was that errors that were accompanied by adequate feedback, hinting at the error source, produced less reactance in the participants than errors that were accompanied by feedback, not hinting at the error source [5].

\subsection{Obstructions}

Obstructions are undesired system prompts or interface changes that stop or inhibit a user in his or her productive workflow. Obstructions are obviously related to System Errors, however as System Errors are usually undesired by developers, whereas obstructions are sometimes implemented on purpose, we decided to treat them separately form System Errors. Edwards et al. investigated the effects of pop-up ads on websites in a two by two by three factorial experiment. They compared the impact of pop-up ads in different situations, e.g. when users viewed content webpages or were having a pause [4]. They found that users regarded pop-up ads as more irritating and intrusive when viewing web pages in a goal-oriented manner, e.g. when they wanted to find a specific information. Edwards et al. concluded that pop-up ads are perceived as more intrusive and irritating when shown while task-oriented web surfing. They conclude that pop-up ads can be regarded as intrusive and irritating and can thereby cause avoidance behavior [4], which can be interpreted as reactant behavior.

\subsection{Reduced Freedom of Choice}

Reactance theory states that people get reactant when they encounter a threat to their freedom of choice $[1,2]$. Therefore, a reduction of a users freedom of choice can lead to those users getting reactant. We found one paper that investigated this effect. Murray and Häubl employed a classical reactance 
paradigm to investigate the effect of diminished freedom of choice of user interfaces on users' reactance. In their experiment, two groups of users could either choose between two types of interfaces or were assigned one particular interface. In a follow-up task, the same participants had to complete a task with a third interface that was found to be superior in terms of ease of use and task completion times in a prestudy. Finally, the participants had to choose from either the interface that was used in the first part of the experiment or the superior interface [14]. Their analysis showed that participants who were free to choose one interface in the first part had a stronger preference for that interface compared to participants who were assigned to a particular interface. Murray and Häubl interpret these result as evidence that a reduced freedom of choice between interfaces can result in reactance [14].

\section{SURVEY}

\subsection{Research Question}

Several causes and triggers for reactance from different domains within human-human interaction are known. A literature search revealed seven of such causes. However, all of the above-mentioned studies were laboratory experiments with fine-tuned and controlled conditions that often were designed to specifically trigger reactance at different intensities. Therefore, a qualitative survey was conducted and partcipants were asked to describe experiences of reactance in real life situations in the past. These collected reports should answer two questions:

A.

Are the causes of reactance that have been identified in the literature search also common in real life situations?

$B$.

What other causes of reactance are relevant for real life situations that may have not been investigated in experimental studies, so far?

\subsection{Participants}

21 persons participated as a convenience sample in the study, all of which were students who participated in a lecture about usability engineering. Gender and age of the participants were not accessed.

\subsection{Procedure}

All participants received an introduction on the topic of psychological reactance in the form of a twenty-minute oral presentation. The presentation included a detailed description of the construct of psychological reactance according to the intertwined model [3] and several examples on when reactance can take place and how it can be measured. At a later point, all participants were asked to describe a situation in which they have experienced reactance in the context of
Table 2: Clusteres and frequency of different causes for reactance that were assessed in the survey.

\begin{tabular}{lc}
\hline Cause & Frequency \\
\hline Reduced Freedom of Choice & 4 \\
Behavioral Restriction & 4 \\
System Errors & 4 \\
Scarceness & 3 \\
Perceived Effort & 2 \\
False Information & 1 \\
Privacy Violations & 1 \\
Undesired Behavior of Device or Service & 1 \\
\hline
\end{tabular}

HCI in the past. The situation descriptions were collected in a hand-written survey. Later on, all responses were transcribed manually and pseudonymized. Due to a false understanding of the task, one response was not considered for further analysis. In the analysis, we first extracted the described causes for reactance for each individual description. Afterwards, those extracted causes we grouped into seven different clusters of similar items. This clustering served as a normalization for the identified reactance causes. The steps of extracting and clustering the reactance causes were performed by both authors separately. In a last step, discrepancies between the two sets of clusters were discussed and resolved. The final clusters were then named. A final list of the causes identified in the survey is shown in Table 2.

\subsection{Results}

In total, eight different causes for reactance in the context of human-computer interfaces could be identified. The categories are described in the following.

3.4.1 Reduced Freedom of Choice. Reduced freedom of choice was mentioned as the cause of reactance in four separate responses to the survey. Reduced freedom of choice describes a situation in which users are confronted with a number of options of which none is desired. This is different from a classical dilemma, because in the described situations, desirable alternatives would have been possible. However, such alternatives simply have not been presented. One example from the survey is the update function of windows, which can be postponed either by 10 minutes, one hour or four hours, but which cannot be canceled or postponed to a custom time. In another example, one payment option was removed by an online shop. The participant claimed not to have bought anything in that show since then.

3.4.2 Behavioral Restriction. Behavioral restiction as a cause for reactance is a visible restriction or denial of certain system functions. In an example from the survey, one participant who is a computer scientist described that he or she got a laptop from his or her employer but was not granted admin rights to install a program. The participant claimed that this was additional motivation to bypass the restriction until he or she found a program that did not need to be installed. 
In another example, a participant used a shareware that limited its functionality after a certain period of time, as a consequence, the participant pirated the program (...instead of purchasing it or using it with the limited functionality).

3.4.3 System Errors. Devices or services are not always operating as they are intended to. The category of system errors as a cause for reactance do include malfunction due to technical errors or limitations but also include bad (in the eyes of the user) interaction design. One example from the collected dataset mentions an incompatible application that dos not start on the participant's smartphone. Another response is an example for a technical limitation rather than a classical error. The participant reported that he or she got reactant when the smartphone battery was at about $5 \%$ in an important situation but still shut down. We choose to include this example in the cluster system errors, however one could easily argue for it belonging to behavioral restrictions or false information (inaccurate information), as well.

3.4.4 Scarceness. This is a special case in the current study because the impression of scarceness is usually not a mistake in the design but is done on purpose. Scarceness in often used in Marketing to make a product appear more desirable. Three students reported that they bought products because of perceived scarceness of those products. Especially online shops have a variety of ways to make products appear scarce. For example, they can display (or fake) the number of articles that are still in stock. Out of the three reports about scarceness, two indicated that the purchase was regretted at a later point, but there was no indication of a cancellation or retour of the order.

3.4.5 Perceived Effort. This describes a situation in which the effort to complete a task is perceived as unnecessarily high. One report described a situation in which an important function was implemented in such a way that it was hard to find even after multiple uses of the respective website. Another participant claimed that he or she was repelled by a long explanatory text before he or she could connect to a network and that he or she did not connect to that network for month because of that explanatory text. That person also did not want to just accept the terms that were stated in the text without reading it.

3.4.6 False Information. One person mentioned that false information triggered reactance. False information means that an interface provides information that later turns out to be false. The example from the dataset states that a software update which was supposed to take 10 minutes, according to the dialogue, took more than 30 minutes and was still not finished. The person claimed to have cancelled the update after 30 minutes and wanted to postpone it to some later point.

3.4.7 Privacy Violation. Privacy violation as a reason for reactance was mentioned once. In the report, the participant claimed that he or she learned that a messenger service was analyzing personal information from messages and images and was even giving away those information to third parties. The participant claimed to have moved to another messenger service as a consequence.

3.4.8 Undesired Behavior or Device or Service. This means that the device or service acts in a way that the user does not want. An example from the dataset is an automatic light that switches itself off too early, what then resulted in the user entering a reactant state.

\section{DISCUSSION}

\subsection{Literature Search}

A search for literature on psychological reactance in the context of human-computer interfaces found ten studies in which the topic of psychological reactance was directly investigated or at least possible causes were discussed. Seven different causes of reactance were identified among those ten studies (see Table 1). The domains in which the studies were performed ranged from emails to smart homes and artificial agents and therefore covered a wide area of human-computer interaction, suggesting that the topic of reactance is relevant for the whole field.

\subsection{Survey Discussion}

A qualitative survey was conducted as an addition to the literature search, 21 results were collected. 20 of those reports were analyzed according to what situations within humancomputer interaction can trigger reactance. As a result, eight different causes of reactance in human-computer interaction were extracted.

Research Question A. Research question A asked whether the causes of reactance that were found in the literature search were also common in real life situations. The survey showed a mixed result. Three of the seven causes that were found in literature were also mentioned in the survey. However, four causes of reactance from the literature were not mentioned in the survey, see Table 3 for an overview.

Research Question B. The second research question asked about other causes of reactance in real life situations. The survey was quite fruitful in this aspect. We could identify five causes of reactance from real life situations that have not yet been addressed in human-computer interaction literature. However, one of which, scarceness, is a common tactic in online marketing. Three students mentioned the marketing method of emphasizing scarcity of their products to use reactance for selling products. The impression of scarcity can persuade users to buy products as part of reactant behavior. The freedom threat in this case is the anticipation of unavailability of the product. The reactant behavior of avoiding that freedom threat by buying the (perceived) scarce product then avoids that threat. Scarceness in marketing was part of the introduction to reactance that the participants received prior to the survey, so it is no surprise that scarceness was mentioned. It is however interesting that out of those three, two wrote that they regretted making the purchase. This 
is an interesting result in this context because it is worth investigating if this might not turn out to have negative consequences for the retailer in the long term.

4.2.1 Criticism. The survey design has several weaknesses. The first of which is that all participants were experts in human-computer interaction and received an introduction to the concept of psychological reactance before. This probably resulted in a bias towards the situations that were reported. However, this survey design was used, because it removed the necessity of conducting guided interviews which would have resulted in a lot more effort. Also, the bias from the prior reactance introduction would probably just have been replaced by the bias of the reactance knowledge of the interviewing person. The aim of the survey was to identify more probable causes or reactance in the human-computer interaction context. The bias that was introduced by the survey design probably resulted in a higher number or stereotypical causes being reported, such as situations in which personal freedom the threatened (Reduced Freedom of Choice and Behavioral Restrictions are indeed under the three causes that have been mentioned the most). However also the appearance of system errors was mentioned four times and this cause cannot simply be deduced from traditional reactance theory. Another result of such a bias would be less diverse responses. A smaller number of identified causes is a worse effect than an unrepresentative frequency distribution of the identified causes. However, this does not reduce the validity of the identified causes and is therefore tolerable. The same is true for the task description that was used. We only asked each participant to report "a" situation in which they experienced reactance. This does not exclude multiple responses in general, but there was no participant who reported more than one situation. This is therefore another potential source of improvement for follow-up studies.

\subsection{Implications for Developers and Designers}

The previous sections introduced a variety of causes for reactance. Fortunately, there are ways to reduce and moderate reactance, too. In the following, some means of easing the reactance effects for the different causes are provided.

Privacy Violation. White et al. [17] and Lee et al. [12] both found causes of reactance that were concerned with collecting of personal information [12] or inappropriate personalization on e-mails [17]. White et al. claim that reactance to personalization occurs when users get the impression of being identifiable or observable by the sender (a company). Also, the survey revealed a situation in which privacy violation by a messaging service induced reactance. The person who reported the situation also mentioned to have migrated to another provider because of the privacy violation, showing how severe consequences of privacy violations can be for a provider. In their paper, White et al. also found that reactance was moderated by the appropriateness of the requested personal information [17]. This means that collecting personal information will make users particularly reactant if there is no obvious reason why such information is collected. If for example a flashlight app asks for permission to access the contacts of the smartphone, users might get reactant because they assume some wrongdoing by the developers. On the other hand, an email client probably will not face such risk because the required information is well justified.

Reduced Freedom of Choice. User reactance caused by reduced freedom of choice was investigated by [14]. They found that users can become reactant if their options are reduced. The survey included two similar reports, where participants described reactance as a result of reduced options to choose from. Brehm states that reactance can be triggered by a freedom threat (reduced freedom of choice) [1,2]. Therefore it might be inevitable that users become reactant if they know that their freedom of choice has been reduced. One report from the survey also mentioned a situation of reduced freedom of choice where Windows produced a pop-up menu in which a restart was requested but the options only included several options of postponement. An option for custom postponement or cancellation until manual restart would have easily handed back the impression of control to the user and thereby avoided reactance.

System Errors. Literature search found one study that dealt with system errors and their implications for reactance. Ehrenbrink et al. claimed that inadequate feedback on system errors can cause reactance [5]. Also, the survey included two reports in which system errors resulted in reactance. Suggesting to keep malfunctions and errors should at a minimum is futile, because every developer will do that, anyway. However, the findings underline the importance of quality control for Devices and Services. Also, error handling should be implemented thoroughly, Ehrenbrink et al. reported that an adequate feedback that helps users to understand the cause of the error can help reducing the reactance effects [5].

Persuasive attempts. Reactance as a result of persuasive attempts was investigated in three of the papers that were identified by the literature search. All papers claimed that persuasive attempts by a system can result in users getting reactant $[11,13,15]$. Persuasive attempts are not only common in recommender systems but in a less strong manner also in introduction functions of newly installed software or devices. The complexity and variety of technology in our everyday life prohibits users to become experts of every technological product that they use. This gives rise to the need of developers and service providers to aid users in their decision making and handling of their devices and services. Usually, this is done via introduction functions or messages that guide users through their first interaction with a product. While such introduction sessions may proof beneficial for users most of the time, it can nevertheless be perceived as a persuasive attempt and thereby cause reactance effects. We therefore suggest employing the "but you are free to accept or refuse" technique by Gueguen et al. [8]. They found that underlining a persons' freedom by adding a hint, such as "but you are 
free to accept or refuse" can reduce reactance effects [8]. In the case of introductory functions this could mean to include a skipping option or simply to ask users if they really want an introduction session.

Self-Adaptation. Adaptivity was not mentioned in the survey but there is a report in literature where reactance effects of adaptive system behavior were found [6]. As for previous causes of reactance, we also suggest underlining the users freedom of choice or the users control over the system during interaction to reduce possible reactance effects. In the case of adaptive or proactive systems this can be done by adding customization or by providing meaningful information on how or why an action was performed by the system.

Forced Response. Stieger et al. reported in their paper [16] that forced response to online surveys can result in reactance of the participants. This reactance cause is related to the report from the survey (which was ultimately grouped in the category Reduced Freedom of Choice) where windows only provided different time options for an upcoming restart but did not provide an option to avoid that restart. Especially in research it is often desired to collect complete datasets of questionnaires. Forced response might be needed more in electronically answered questionnaires than in paper questionnaires because users often unintentionally fail to correctly fill out checkboxes or radio buttons. In those cases, we suggest not to enforce complete datasets by forced response but to implement a message if some boxes remain unchecked, together with an option to leave those items unanswered if needed. This way, online surveys will still resolve the problem of accidentally missed items but will not force participants into something that they do not want.

Obstructions. Obstructions as a cause of reactance have not been mentioned in the survey but have been discussed in literature. Edwards et al. found that obstructions by pop-up adds can result in reactance, especially when they are perceived as severe obstructions from e.g. productive work [4]. We therefore propose to implement some context awareness into systems, if possible. Messages that are not vital or adds can then be displayed when the user is not in a highly demanding task. This way, the user will get less reactant and e.g. adds might be more successful. Also, messages by other programs could be opened in the background so that users are not immediately interrupted in the task that they are doing.

Behavioral Restrictions. Behavioral restrictions were reported in four responses from the survey but have not been investigated in the reviewed literature. In one report, a shareware reduced the feature set after a trial period and still displayed the functionalities in the menu. Reactance can result in people doing the opposite of what the message intended to counteract the perceived freedom threat [3]. This also happened in the reported situation. Because of the blocked features, the user turned reactant and did not buy the shareware. Instead, the user pirated it. We therefore suggest that developers try to avoid reactance reactions of their users, especially in the case of trial software, by hiding the options that are not available until purchase. Even though showing the purchasable features can make users wanting to access those features more, a reactance reaction might lead to pirating those features instead of buying them, as the report from the survey suggested.

Scarceness. Scarceness was not investigated in the papers from human-computer interaction that we review, but scarceness is a commonly used effect in marketing. As scarceness is often applied intentionally, we will not discuss how to avoid it at this point. Nevertheless, scarceness was mentioned in the reports from the survey three times. Two of which, it was added that the person later regretted the purchase. It is surely worth investigating if the products that were purchased because of scarceness-based marketing might be returned more often than products that were purchased because of other reasons.

Perceived Effort. Two of the reports from the survey mentioned perceived effort as a cause of reactance. Unnecessarily high effort is probably a bigger problem in productive scenarios, rather than in gaming scenarios. Avoiding unnecessarily high effort should be the goal of every designer and developer, anyway. Task efficiency is even given as part of the definition of usability in ISO 9241-11 [10].

False Information. False Information was mentioned once in the survey. In the report, the user got reactant when an update that was reported to take ten minutes was still not complete after 30 minutes. Developers should not assume that users are always aware that e.g. given times for completion of a task or remaining battery are merely an estimation. If information in dialogues is uncertain, this should somehow be marked. A possible way of doing so is to give a time interval instead of a precise number. Otherwise, users cold expect the provided estimation to be exact and then turn reactant according to the perceived importance of lost time or other resources due to the imprecise information.

Undesired Behavior or Devices and Services. According to one survey report, undesired behavior of the device or service resulted in reactance. Undesired behavior is probably mostly an issue in proactive systems or systems that are used for the first time, as otherwise users know what to expect when they use a device or service. Possibly, it is the discrepancy between the expected behavior and the actual behavior of the device which causes reactance. Thus, to avoid reactance, differences in the mental model of the device between the user and the designer should be kept at a minimal level. Also, proactive systems should implement features feedback mechanisms that allow users to anticipate and ideally to influence their behavior.

\subsection{General Discussion}

There is little overlap between the causes of reactance in literature and the causes of reactance that were identified in the survey. In fact, the only commonly mentioned causes 
are privacy violations, reduced freedom of choice and system errors. A possible explanation for this is that in literature, experiments that are concerned with reactance often chose one of the classical stimuli, such as persuasive attempts (interaction with persuasive systems) and then vary the strength of some moderating variable $[4,15,17]$.

Table 3: Clusteres of different causes for reactance that were assessed in literature and the survey.

\begin{tabular}{lcc}
\hline Cause & Literature & Survey \\
\hline Privacy Violations & $\checkmark$ & $\checkmark$ \\
Reduced Freedom of Choice & $\checkmark$ & $\checkmark$ \\
System Errors & $\checkmark$ & $\checkmark$ \\
Persuasive Attempts & $\checkmark$ & $x$ \\
Self Adaptation & $\checkmark$ & $x$ \\
Forced Response & $\checkmark$ & $x$ \\
Obstructions & $\checkmark$ & $x$ \\
Behavioral Restriction & $x$ & $\checkmark$ \\
Scarceness & $x$ & $\checkmark$ \\
Perceived Effort & $x$ & $\checkmark$ \\
False Information & $x$ & $\checkmark$ \\
Undesired Behavior of Device or Service & $\boldsymbol{x}$ & $\checkmark$ \\
\hline
\end{tabular}

\section{CONCLUSION AND FUTURE WORK}

To the authors knowledge this work is the first collection of causes for reactance in the context of human-computer interaction. There are probably more causes of reactance that are not covered by the list provided here and a larger and systematic approach for researching reactance in humancomputer interaction is needed. Four directions for further research are therefore proposed. First, a systematic survey of the frequency of causes for reactance to find out, what are the most common causes should be conducted. Second, it is not guaranteed that each cause can be applied to all or multiple domains. It is for example quite possible that perceived effort is a big cause of reactance in productivity software but does not play any role in gaming. Third, this survey did not address effect sizes of the reviewed empirical studies. Effect sizes are however important clues on the relevance of the reactance causes under investigation. Such information would be interesting and valuable for developers and designers in the field of human-computer interaction. Fourth, in practice, multiple causes for reactance can appear together, e.g. perceived effort and false information. The survey resulted in a report where an update took longer than indicated before. This is obviously a case where false information was provided. But if that update also prohibits the user from an important or urgent task, it might also mean more effort or stress in the future. The question is, how multiple causes influence the level of the reactance reaction. The literature review and the survey have accumulated a list of twelve causes of reactance. Developers should take these into account to reduce reactance of their users and perhaps to avoid them smashing their products against their desks.

\section{ACKNOWLEDGEMENTS}

This work was supported by Bundesministerium fr Bildung und Forschung - BMBF, Software Campus, grand no. 01IS12056, Sozialpsychologische Aspekte von Smart Homes (SPASH).

\section{REFERENCES}

[1] Jack Williams Brehm. 1966. A Theory of Psychological Reactance. Academic Press, New York.

[2] Sharon S. Brehm and Jack Williams Brehm. 1981. Psychological reactance: A theory of freedom and control. Academic Press New York.

[3] James Price Dillard and Lijiang Shen. 2005. On the Nature of Reactance and its Role in Persuasive Health Communication. Communication Monographs 72, 2 (2005).

[4] Steven M Edwards, Hairong Li, and Joo-Hyun Lee. 2002. Forced exposure and psychological reactance: Antecedents and consequences of the perceived intrusiveness of pop-up ads. Journal of Advertising 31, 3 (2002), 83-95.

[5] Patrick Ehrenbrink, Xin Guang Gong, and Sebastian Möller. 2016. Implications of Different Feedback Types on Error Perception and Psychological Reactance. In Proceedings of the 28th Australian Conference on Computer-Human Interaction (OzCHI'16). ACM, New York, NY, USA, 358-362. https://doi.org/10.1145/3010915. 3010994

[6] Patrick Ehrenbrink, Stefan Hillmann, Benjamin Weiss, and Sebastian Möller. 2016. Psychological Reactance in HCI: A Method Towards Improving Acceptance of Devices and Services. In Proceedings of the 28th Australian Conference on Computer-Human Interaction (OzCHI '16). ACM, New York, NY, USA, 478-482. https://doi.org/10.1145/3010915.3010978

[7] Google Inc. 2004. Google Scholar. (2004). Retrieved March 15, 2017 from https://scholar.google.com/.

[8] Nicolas Guéguen and Alexandre Pascual. 2005. Improving the response rate to a street survey: an evaluation of the" but you are free to accept or to refuse" technique. The Psychological Record 55, 2 (2005), 297.

[9] "IlluminatiPulskie" via Wikimedia Commons. 2016. Angry German Kid.jpg. (2016). Retrieved March 17, 2017 from https: //commons.wikimedia.org/wiki/File:Angry_German_Kid.jpg.

[10] ISO 9241-11. 1999. Ergonomische Anforderungen fr Brottigkeiten mit Bildschirmgerten Teil 11: Anforderungen an die Gebrauchstauglichkeit - Leitstze. (Januar 1999).

[11] Matthias Laschke, Sarah Diefenbach, Thies Schneider, and Marc Hassenzahl. 2014. Keymoment: Initiating behavior change through friendly friction. In Proceedings of the 8th Nordic Conference on Human-Computer Interaction: Fun, Fast, Foundational. ACM, 853-858.

[12] Gyudong Lee and Won Jun Lee. 2009. Information \& Management Psychological reactance to online recommendation services. Information \& Management 46, 8 (2009), 448-452.

[13] Shenghua Liu, Sacha Helfenstein, and Ari Wahlstedt. 2008. Social Psychology of Persuasion Applied to Human Agent Interaction. (2008).

[14] Kyle B. Murray and Gerald Häubl. 2011. Freedom of Choice, Ease of Use, and the Formation of Interface Preferences. MIS Quarterly 35, 4 (2011), 955-976.

[15] Maaike Roubroeks, Jaap Ham, and Cees Midden. 2011. When Artificial Social Agents Try to Persuade People: The Role of Social Agency on the Occurrence of Psychological Reactance. International Journal of Social Robotics 3, 2 (2011), 155-165.

[16] Stefan Stieger, Ulf-Dietrich Reips, and Martin Voracek. 2007. Forced-response in online surveys: Bias from reactance and an increase in sex-specific dropout. Journal of the American society for information science and technology 58, 11 (2007), 1653-1660.

[17] Tiffany Barnett White, Debra L Zahay, Helge Thorbjørnsen, and Sharon Shavitt. 2008. Getting too personal: Reactance to highly personalized email solicitations. Marketing Letters 19, 1 (2008), $39-50$. 